\title{
It Does Not Fitts My Data! Analysing Large Amounts of Mobile Touch Data
}

\author{
Niels Henze and Susanne Boll \\ University of Oldenburg, \\ Escherweg 2, 26121 Oldenburg, Germany \\ \{niels.henze, susanne.boll\}@uni-oldenburg.de
}

\begin{abstract}
Touchscreens are the dominant input device for smartphones and learning about smartphone users' touch behaviour became even more important. We developed a game for Android phones to collect a truly large amount of touch data from diverse devices and players. A part of the game is designed as what we expected to be a Fitts' law task. By publishing the game in the Android Market we collected 5,359,650 micro tasks from 63,154 installations of the game. Using Fitts' law to find a model for these tasks we found a very weak correlation and an implausible high index of performance across different devices. Further analysis shows a similar correlation between time and distance as with Fitts' law but only a very weak correlation with the targets' width.
\end{abstract}

Keywords: Fitts' law, mobile phone, touch screen, app store, large-scale.

\section{Background}

Fitts' law [2] is a model of human movement that predicts the time required to rapidly move to a target. It describes the correlation between the distance to a target and it's width with the time to reach the target. [3] describes Fitts' law as "one of the most robust, highly cited, and widely adopted models to emerge from experimental psychology" [3]. The index of performance (IP), that is determined using Fitts' law, enables to compare different input devices and is even proposed by ISO 9241-9 [1] for this purpose. Fitts' law has been applied to a wide range of devices, including mouse, touchpads, and trackballs [3]. The IP was used to compare these devices and we assume that the IP can also be used to assess the quality of smart phones' touch screens.

To collect data from a number of different devices, in a natural usage context, and a sufficient number of participants we developed a game and published it in the Android market. The game requires touching targets and records the players' behaviour. A part of the game is a task that requires to sequentially tapping simultaneously presented circles. Using data from 63,154 installations of the game and considering tapping each but the first of the simultaneously presented circles as a task we collected 5,359,650 tasks.

\section{Collecting Touch Data in the Large}

The gameplay of Hit It! is inspired by the task used by Park et al. [4]. Circles are displayed on the screen (see Figure 1) and the player has to touch these targets. Each level consists of multiple micro levels. Different target sizes are used in each level. In 
most micro levels, one circle is presented to the player. The player advances directly to the next micro level as soon as the target is hit. If a target has not successfully been hit in a certain time frame it is counted as a miss. Every fifth micro levels consist of multiple simultaneously presented targets. As soon as a target is successfully hit it disappears. The player must hit all targets to advance to the next micro level. To make a game out of the two basic tasks the player must complete a micro level in a certain timeframe. The time is reduced from micro level to micro level while the player proceeds through a level. The player receives a penalty point if a target has not been hit and the game is lost when the player collected three penalty points. The faster players hit the targets the more scores they get.
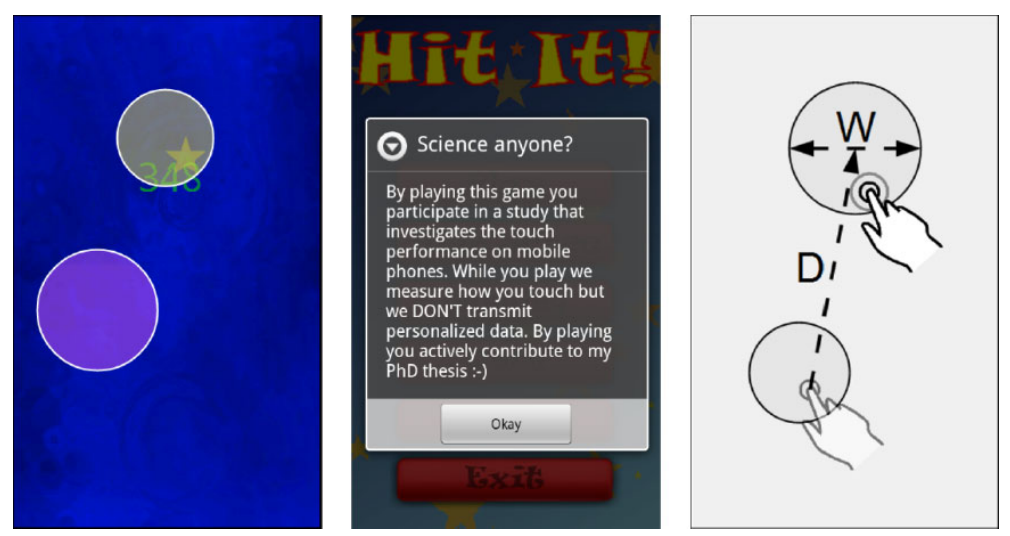

Fig. 1. In-game screenshots of the game (left), information presented to the player when the game is started (centre), and how W \& D are derived for Fitts' law (right)

We do not collect data that allows identifying individual players or installations. We still decided to clearly inform players about the fact that data is collected in order to act ethically. A modal popup (see Figure 1) shown when the game is started for the first time tells the players that data is collected for research purpose while the game is played. Data about the used device and the performance of a player is collected by transmitted the data to our server. A unique identifier for each installation is derived by hashing the device's "Android ID". We also collect the user's locale (e.g. "en_GB") and the device's type (e.g. "GT-I9000" for Samsung Galaxy S). Most importantly, we record the position and size of the targets for each micro level. We also record the position of each touch event and the time elapsed since the start of the micro level. We published Hit It! in the Android Market on October 31, 2010. Till April 04, 2011 the game was installed 192,250 times $^{1}$.

\section{Applying Fitts' Law}

When developing the gameplay we designed two tasks. The first task is just tapping a single target but the second task was designed to be a Fitts' law task. In the following

\footnotetext{
${ }^{1}$ Number of installs according to Google's Developer Console.
} 
we analyze the data from the second task using Fitts' law and investigate the correlation of movement time with the target's distance and width afterwards.

\subsection{Correlation and Throughput}

In order to apply Fitts' law we use the Shannon formulation $M T=a+b \cdot \log _{2}\left(1+\frac{D}{W}\right)$ proposed by [3]. We only consider micro levels where multiple circles are presented simultaneously, the device is held in an upright posture (according to the phone's accelerometer), and the level's error rate is below 4\%. When the player hits the first circle the touched position and the current time is used as the start for the first task (see Figure 1). MT is the time that elapsed until the second circle is hit. D is the distance from the position of the first tap to the centre of the second circle and $\mathrm{W}$ is the diameter of the second circle. Analogical we proceeded with the other targets using the touched position that hit one circle and the according subsequent circle. Thereby multiple tasks are derived from a single micro level.

With the described approach we determined 5,359,650 tasks from 63,154 installations. Using linear regression we determined a (intercept) and $\mathrm{b}$ (slope) and found that $a=.200$ and $b=.040$. The correlation of the data with the resulting formula is $r=.14$. The index of performance $\left(I P=\frac{1}{b}\right)$ is $\mathrm{IP}=25.01$. As the determined IP is implausibly high (highest IP reported by [3] is 13.7 for an eye tracker) and the correlation is very weak ([3] reports correlations between .70 and .99) we selected the considered data more carefully. Using only data provided by 4,873 installations running on a Samsung Galaxy S (292,515 tasks), the most common device in our dataset, results in $a=.207$, $\mathrm{b}=.029$, and $\mathrm{r}=.13$. With 34.57 the IP is still implausibly high. Considering only tasks from one particular level (992 installations, 5,328 tasks) results in $a=.176, b=.060$, $\mathrm{r}=.33$, and $\mathrm{IP}=16.55$.

One reason for the unsatisfying correlation might be the small screen size of the Samsung Galaxy S (4.0in diagonal size) that does not require moving the hand but only one finger. Therefore, we repeated the analysis for the Samsung Galaxy Tab (352 installations, 2,134 tasks) that has a screen with 7.0in. Again only tasks from one level are considered. Using linear regression we determined intercept and slope and found $a=.165$ and $b=.063$. The correlation of the resulting formula is $r=.33$ and the index of performance is IP=15.80. Regardless of the way we constrain the dataset, the IP is unlikely high and the correlation is weak for all tested devices. While the high IP only suggest that the used task might be different from what is commonly used as Fitts' law task, the rather low correlation shows that Fitts' law is not a good model for the used tasks.

\subsection{Correlation with Targets' Width and Distance}

In order to investigate why Fitts' law cannot be used as a model for our data we analyzed the data in more detail. We tested the correlation with the target's width and the distance to the target independently. For a true Fitts' law task one would expect that there is at least a weak correlation with both aspects that are composed in Fitts' law. As the measurement unit is not crossed out when looking at width and distance separately we have to treat each device independently. 
Using again the data from one level provided by the Samsung Galaxy S (992 installations, 5,328 tasks), the correlation between time and distance is $r=.33$. Compared with the correlation using Fitts' law (also $r=.33$ ) the difference is very small. This suggests that most of the variance that can be explained by the correlation with ID can also be explained by the correlation with the distance to the target. Determine the Pearson correlation between time and width using the same dataset results in $\mathrm{r}=.11$. Testing different functions (e.g. $\log _{2}(\mathrm{~W}), 1 / \mathrm{W}, \log _{2}(1 / \mathrm{W})$ ) does not reveal a higher correlation.

\section{Discussion and Future Work}

Analyzing the data that resulted from players that touch a sequence of circles we found only a weak correlation when applying Fitts' law as a model for the data. Furthermore, the IP is implausible high. Further looking at the data we found a similar linear correlation between time and distance as if using Fitts' law. In contrast we found a much smaller correlation between time and the width of the target. Our results suggest that, for our task, the time required to hit a target partially depends on the distance to the target. A target's width, however, has a much smaller effect on the time required to hit a target.

The results presented in this paper are only a fraction of what can be investigated using the collected data. Further analysis showed that touch positions are systematically skewed and a compensation function that shifts the users' touches can reduce the error rate [5]. An area we consider interesting for future work is investigating touch sequences. How a target is hit might not only depend on the target's size and location but also on preceding touch contacts and the elapsed time. Applying such findings might result in a function that shifts the touch events to improve the users' touch precision that in generally applicable. We would like to share the data with other researchers to enable further analysis ${ }^{2}$.

\section{References}

1. Douglas, S., Kirkpatrick, A., MacKenzie, I.: Testing pointing device performance and user assessment with the ISO 9241, Part 9 standard. In: Proc. CHI (1999)

2. Fitts, P.M.: The information capacity of the human motor system in controlling the amplitude of movement. Journal of Experimental Psychology 47 (1954)

3. MacKenzie, I.: Fitts' law as a research and design tool in human-computer interaction. Human-computer Interaction 7(1), 91-139 (1992)

4. Park, Y., Han, S., Park, J., Cho, Y.: Touch key design for target selection on a mobile phone. In: Proc. MobileHCI (2008)

5. Henze, N., Rukzio, E., Boll, S.: 100,000,000 Taps: Analysis and Improvement of Touch Performance in the Large. In: Proc. MobileHCI (2011)

\footnotetext{
${ }^{2}$ The complete dataset can be retrieved from http://nhenze.net/?page_id=673
} 